\title{
Introduction to the Symposium on Practical Aspects of Angiotensin-Converting Enzyme Inhibition
}

\author{
ROBERT J. CHILTON, D.O. \\ Associate Professor of Medicine \\ Director of Electrophysiology \\ University of Texas Health Science Center \\ San Antonio, Texas
}

The development of captopril, the first angiotensin converting enzyme (ACE) inhibitor, is a benchmark in the treatment of hypertension and congestive heart failure. The mode of action of this drug on the renin-angiotensin-aldosterone (RAA) systems offers a totally new, exciting approach to the treatment of these conditions. There is little doubt that the importance of this new class of therapy will surpass that of the rauwolfias in the $1950 \mathrm{~s}$, the diuretics in the $1960 \mathrm{~s}$, and the beta-blockers in the 1970s.

The RAA system is a major pressor mechanism. By inhibiting the enzyme that is responsible for the conversion of angiotensin Ian inactive compound-to angiotensin II, a potent vasoconstrictor, the $\mathrm{ACE}$ inhibitor interferes with the activation of a very potent and specific mechanism that contributes to the maintenance of hypertension. Angiotensin II elevates blood pressure in two ways: directly, by causing contraction of vascular smooth muscle; and, indirectly, by stimulating the adrenal cortex to release aldosterone. With the rise in angiotensin II levels, blood pressure and sodium concentration, the stimuli for renin production diminish. In normotensive persons, the RAA system acts as a self-regulatory, closed-loop feedback system for maintaining circulatory homeostasis of sodium and fluid volume; in hypertensives, the RAA system undergoes pathologic changes that result in sustained and inappropriate activation.

Although a wide variety of antihypertensive therapies have been available over the past 30 years, complications of hypertension are still a leading cause of death. An important obstacle has been the side effects associated with antihypertensive therapies which often make the patient feel worse on treatment than off. A high rate of noncompliance with therapeutic regimens is the usual result: patients would rather risk the complications of hypertension than diminish their quality of life.

The ACE inhibitors offer a new degree of freedom from the side effects usually associated with drug therapy of hypertension. For the typical hypertensive patient, who is usually free of symptoms before initiating treatment, the ACE inhibitors enhance outlook and lifestyle while affording blood pressure control. Data from a landmark study comparing captopril to methyldopa and propranolol substantiate this view. For the first time, quality of life has been evaluated in a systematic, comprehensive and integrated fashion, using objective and reliable assessment instruments. The results of this study demonstrated that all three therapies were effective in lowering blood pressure; however, their impact on quality of life differed significantly. Compared to baseline values, captopril maintained and in several instances enhanced the patient's quality of life.

In addition to its established efficacy in the treatment of hypertension, the ACE inhibitor also offers an important advance in the treatment of congestive heart failure. The RAA system normally functions to help maintain adequate organ perfusion. In patients with congestive heart failure, organ perfusion is compromised due to low cardiac output. The kidney is one of the first organs to be affected. Under conditions of low perfusion, the kidney responds by secreting the enzyme renin, which sets off a series of events that ultimately results in the production of angiotensin II. This contributes to the pathophysiology of congestive heart failure by 
increasing systemic arteriolar resistanceafterload-and by stimulating the secretion of aldosterone, which increases sodium and fluid retention leading to increased preload. The $\mathrm{ACE}$ inhibitors block the reaction between angiotensin I and ACE, thus preventing pressor stimulus. Vascular dilatation occurs with reduction of the impedance to left ventricular ejection. The net effect is improved cardiac output, redistributed perfusion to peripheral tissues and relief of cardiac dilatation. Functional status and symptomatology improve in the majority of patients.

The papers on experience with the angiotensinconverting enzyme inhibitor, captopril, which appear in this supplement, were presented at a symposium held on July 25 and 26, 1986 in Denver, Colorado. I hope the information provided by this outstanding faculty will be helpful to you. 